\title{
Sequencing and Characterisation of Complete Mitogenome DNA for Rasbora hobelmani (Cyprinidae) with Phylogenetic Consideration
}

\author{
H. H. Chung, ${ }^{a}$ *, C. K. A. Kamar ${ }^{a}$, L. W. K. Lim ${ }^{a}$, Y. Liao ${ }^{b}$, T. T. Lam ${ }^{b}$, and Y. L. Chong ${ }^{c}$ \\ ${ }^{a}$ Faculty of Resource Science and Technology, Universiti Malaysia Sarawak, Sarawak, 94300 Malaysia \\ ${ }^{b}$ School of Public Health, The University of Hong Kong, Sassoon Road, Pokfulam, Hong Kong \\ ${ }^{c}$ Department of Science and Environmental Studies, The Education University of Hong Kong, \\ Tai Po Campus, NT, Hong Kong \\ *e-mail: hhchung@unimas.my
}

Received April 30, 2019; revised September 18, 2019; accepted September 23, 2019

\begin{abstract}
The Kottelat rasbora Rasbora hobelmani is a small ray-finned fish categorized under the genus Rasbora in the Cyprinidae family. In this study, the complete mitogenome sequence of $R$. hobelmani was sequenced using two pairs of primers covering overlapping regions. The mitogenome is $16541 \mathrm{bp}$ in length, housing 22 transfer RNA genes, 13 protein-coding genes, two ribosomal RNA genes and one putative control region. Identical gene organisation was spotted between this species and other Rasbora genus members. The heavy strand contains 28 genes while the light strand contains the remaining nine genes. Most protein-coding genes employ ATG as start codon, except for the COI gene, which exploits GTG instead. The central conserved sequence blocks (CSB-F, CSB-E and CSB-D), variable sequence blocks (CSB-3, CSB-2 and CSB-1) as well as the terminal associated sequence (TAS) are conserved in the control region. The maximum likelihood phylogenetic tree revealed the close phylogeny of $R$. hobelmani with $R$. sumatrana, $R$. aprotaenia, $R$. lateristri$a t a$ and $R$. steineri with bootstrap value of at least $99 \%$. This work acts as milestone towards future evolution and population genetics studies of this species as well as the Rasbora genus.
\end{abstract}

Keywords: Rasbora hobelmani, mitogenome, gene arrangement, light strand origin, phylogeny, Borneo

DOI: $10.1134 /$ S0032945220010014

\section{INTRODUCTION}

The Kottelat rasbora Rasbora hobelmani is a small ray-finned fish under the family Cyprinidae. This fish can be distinguished from other rasbora fishes for the presence of dark lateral stripe from its opercle to caudal base, ending in a precaudal spot (Kottelat, 2001). It can grow up the maximum length of $6.0 \mathrm{~cm}$, feeding on insects. The Kottelat rasbora generally inhabits pools of small upland streams and the spawning spot is usually in ponds and rivers (Kottelat, 2001). This rasbora fish can be found distributed across northern Thailand, eastern Myanmar as well as the Mekong basin (Kottelat, 2001).

The Cyprinidae family is the most species rich freshwater fish family, encompassing 11 subfamilies: Barbinae, Leuciscinae, Danioninae, Cultrinae, Squaliobarbinae, Gobioninae, Cyprinninae, Acheilognathinae, Tincinae, Labeoninae and Xenocypridinae. The Rasbora genus belongs to the Danioninae subfamily (Liao et al., 2010). In Malaysia, rasbora fish are commonly known as "Seluang" fish and the terms differs accordingly based on their locality: "Buntong" in Keningau Dusun, "Londoi" in Dusun, and "Buntad" in Orang Sungei (Inger and Chin, 1990). The
Rasbora genus was classified by Brittan (1954) into 8 species complexes: Lateristriata, Caudimaculata, Argyrotaenia, Einthovenii, Sumatrana-elegans, Trisfasciata, Daniconius and Pauciperforata. The cryptic diversity occurs within the Rasbora genus due to their miniature size and very similar morphological traits (Trontelj and Fiš̀er, 2009). For instance, Rasbora paucisqualis has been confused with Rasbora bankanensis due to the similarity in terms of scale count and colour pattern (Siebert, 1997).

The research focus on the members of the Rasbora genus is just picking up its pace with the two of the rasbora fish being put into the limelight in the field of ecotoxicology (Wijeyaratne and Pathiratne, 2006; Lim et al., 2018). Thus far, the mitogenome of the Rasbora members that are available in the GenBank public domain totalled up to 15 species only (namely $R$. borapetensis, $R$. trilineata, $R$. maculata, $R$. argyrotaenia, $R$. pauciperforata, $R$. steineri, $R$. sumatrana, $R$. aprotaenia, $R$. vaterifloris, $R$. heteromorpha, $R$. espei, $R$. cephalotaenia, $R$. sarawakensis, $R$. daniconius, and $R$. lateristriata) (Tang et al., 2010; Chang et al., 2012; Ho et al., 2014; Zhang et al., 2014; Kusuma and Kumazawa, 2015; Kusuma et al., 2017; Lim et al., 2019), leaving the other 87 rasbora species 